\title{
ON THE PERIODIC MOTIONS OF A CHARGED PARTICLE IN AN OSCILLATING MAGNETIC FIELD ON THE TWO-TORUS
}

\author{
LUCA ASSELLE AND GABRIELE BENEDETTI
}

\begin{abstract}
Let $\left(\mathbb{T}^{2}, g\right)$ be a Riemannian two-torus and let $\sigma$ be an oscillating 2-form on $\mathbb{T}^{2}$. We show that for almost every small positive number $k$ the magnetic flow of the pair $(g, \sigma)$ has infinitely many periodic orbits with energy $k$. This result complements the analogous statement for closed surfaces of genus at least 2 AB15 and at the same time extends the main theorem of AMMP14 to the non-exact oscillating case.
\end{abstract}

\section{INTRODUCTION}

Let $(M, g)$ be a closed connected orientable Riemannian surface and let $\sigma$ be a 2-form on $M$. We call $\sigma$ the magnetic form. We denote with $\omega_{g}$ the standard symplectic form on $T M$ obtained by pulling back the canonical symplectic form on $T^{*} M$ via the Riemannian metric and with $\omega_{g, \sigma}:=\omega_{g}+\pi^{*} \sigma$ the twisted symplectic form determined by the pair $(g, \sigma)$, where $\pi: T M \rightarrow M$ is the projection. The Hamiltonian flow on the symplectic manifold $\left(T M, \omega_{g, \sigma}\right)$ associated with the kinetic energy

$$
E(q, v)=\frac{1}{2}|v|_{q}^{2}
$$

is called the magnetic flow of the pair $(g, \sigma)$ and the trajectories of such a flow are called magnetic geodesics. Indeed, this flow models the motion of a charged particle under the effect of a magnetic field represented by $\sigma$. More precisely, let $\nabla$ be the Levi-Civita connection of $g$ and $Y_{g, \sigma}: T M \rightarrow T M$ be the Lorentz force defined by the formula

$$
g_{q}\left(u, Y_{g, \sigma}(q, v)\right)=\sigma_{q}(u, v), \quad \forall q \in M, \forall u, v \in T_{q} M .
$$

A curve $\gamma: \mathbb{R} \rightarrow M$ solves the equation

$$
\nabla_{\dot{\gamma}} \dot{\gamma}=Y_{g, \sigma}(\gamma, \dot{\gamma})
$$

if and only if $(\gamma, \dot{\gamma}): \mathbb{R} \rightarrow T M$ is a trajectory of the Hamiltonian flow of $E$.

Following [Arn61, Equation (1.1) can be equivalently formulated in terms of the geodesic curvature $\kappa_{g}$ of the curve $\gamma$. Indeed, let $\mu_{g}$ be the area form associated with $g$ and let $f: M \rightarrow \mathbb{R}$ be the unique function such that $\sigma=f \mu_{g}$. We call $f$ the density of $\sigma$ with respect to $\mu_{g}$. For all $k>0$, a curve $\gamma: \mathbb{R} \rightarrow M$ with constant speed $\sqrt{2 k}$ satisfies (1.1) if and only if

$$
\kappa_{g}(\gamma)=-\frac{f(\gamma)}{\sqrt{2 k}}
$$

For example, this shows that, when $M=S^{2}=\left\{x^{2}+y^{2}+z^{2}=1\right\} \subset \mathbb{R}^{3}, g$ is the round metric and $\sigma=\mu_{g}$, every magnetic geodesic is supported on the intersection of $S^{2}$ with some affine plane in $\mathbb{R}^{3}$ not passing through the origin.

A central question in the study of the dynamics of magnetic flows is the existence of closed magnetic geodesics. In AMMP14 it is shown that if $\sigma=d \vartheta$ is exact, then for almost every

Date: October 24, 2018.

2010 Mathematics Subject Classification. 37J45, 58E05.

Key words and phrases. Dynamical systems, Periodic orbits, Symplectic geometry, Magnetic flows. 
$k \in\left(0, c_{u}\left(L_{\vartheta}\right)\right)$ the energy level $E^{-1}(k)$ carries infinitely many geometrically distinct closed magnetic geodesics. Here $c_{u}\left(L_{\vartheta}\right)$ denotes the Mañé critical value of the universal cover (see Con06] or [Abb13] for the precise definition) of the Lagrangian

$$
L_{\vartheta}(q, v)=\frac{1}{2}|v|_{q}^{2}+\vartheta_{q}(v) .
$$

One of the research directions undertaken by the authors of this paper is to extend such result to the case of an oscillating $\sigma$.

Definition 1.1. We say that $\sigma$ is oscillating if there exist $q_{-}, q_{+} \in M$ such that $\sigma_{q_{-}}<0$ and $\sigma_{q_{+}}>0$.

We notice that oscillating forms are a natural generalization of the exact ones, since we can think of exact forms as "balanced" oscillating forms, their integral over $M$ being zero. We already showed in AB15, that the result proved in AMMP14 for exact forms extends to oscillating forms when $M$ has genus at least 2 and $c_{u}\left(L_{\vartheta}\right)$ is replaced by some $\tau_{+}^{*}(g, \sigma) \in\left(0, c_{u}\left(L_{\vartheta}\right)\right]$ (observe that $c_{u}\left(L_{\vartheta}\right)$ is still well-defined since the lift of $\sigma$ to the universal cover is exact). Implementing ideas contained in [AB16], we are now able to treat the case in which $M=\mathbb{T}^{2}$ is the two-torus. After we submitted our manuscript, the case of the two-sphere has also been solved by the authors in collaboration with Abbondandolo, Mazzucchelli and Taimanov $\mathrm{AAB}^{+} 16$.

The aim of the present paper is therefore to prove the following

Theorem 1.2. Let $\sigma$ be an oscillating 2 -form on $\left(\mathbb{T}^{2}, g\right)$. There exists a positive real number $\tau_{+}(g, \sigma)>0$ such that for almost every $k \in\left(0, \tau_{+}(g, \sigma)\right)$ the energy level $E^{-1}(k)$ carries infinitely many geometrically distinct closed magnetic geodesics.

Remark 1.3. A generic 2-form $\sigma$ on $M$ is either oscillating or symplectic. The latter case has also been object of intensive research in relation with the existence of periodic orbits. Indeed, the following facts are known when $\sigma$ is symplectic. If $M \neq S^{2}$ there exist infinitely many closed magnetic geodesics on every low energy level [FH03, GGM15]. If $M=S^{2}$ there are either two or infinitely many closed magnetic geodesics for every low energy level [Ben16]. Under some non-resonance conditions the second alternative holds for every low energy level [Ben]. However, there are also examples of magnetic systems with a "low" energy level having exactly two closed magnetic geodesics [Ben15].

Remark 1.4. In AM16, Theorem 6.1] the existence of local minimizers has been extended to the case of magnetic Tonelli systems $(L, \sigma)$ for energies belonging to the interval $\left(e_{0}(L), e(L, \sigma)\right)$, see AM16] for the precise definitions. This yields a generalization of Theorem 1.2 to that setting, as explicitly stated in [AM16, Theorem 6.2]. Very little is known on periodic orbits for systems that are not Tonelli. A result asserting the existence of periodic orbits for almost every compact regular energy level of a general Hamiltonian system on $S^{2}$ was recently obtained in [BZ15].

The proof of Theorem 1.2 relies on a variational characterization of closed magnetic geodesics with energy $k$. They correspond to the zeros of a suitable closed 1-form $\eta_{k}$, called the action 1-form, defined on the Hilbert manifold $\mathcal{M}$ of $H^{1}$-loops with arbitrary period. The main difference with respect to the case " $\sigma$ exact" in AMMP14, or with the case " $M$ has genus at least 2 " in AB15, is that, if $\sigma$ is a non-exact form on $\mathbb{T}^{2}$, then $\eta_{k}$ cannot be described as the differential of a functional on the subset of non-contractible loops. Work of Taumanov shows that we cannot circumvent this problem by simply restricting the study to the subset of contractible loops. Indeed, he recently constructed some concrete examples of oscillating magnetic forms on $\mathbb{T}^{2}$ for which the orbits given by Theorem 1.2 cannot be contractible [Tai15]. 
The lack of a globally defined functional gives rise to two main difficulties. First, we cannot use the level sets of the functional to distinguish between different magnetic geodesics (this was an essential point for the arguments of [AMMP14]). As we explain below, we solve this problem exploiting the fact that the fundamental group of $\mathbb{T}^{2}$ is torsion-free. Second, we need to find a class of sequences $\left(\gamma_{h}\right)_{h \in \mathbb{N}} \subset \mathcal{M}$, generalizing the classical Palais-Smale sequences, and for which a compactness theorem holds. This second problem is solved by making use of recent work of the authors. Indeed, in [AB16, Theorem 2.1] we showed that every vanishing sequence $\left(\gamma_{h}\right)$ (i.e. a sequence such that $\left|\eta_{k}\left(\gamma_{h}\right)\right| \rightarrow 0$ ) whose periods are bounded and bounded away from zero admits a converging subsequence. Since limiting points of vanishing sequences are zeros of $\eta_{k}$, the aforementioned compactness property provides the right tool to prove the existence of closed magnetic geodesics with energy $k$.

The starting point of the proof of Theorem 1.2 is the existence of a zero $\alpha_{k}$ of $\eta_{k}$ of a particular kind, namely a "local minimizer" for $\eta_{k}$ (see Section 4 for a precise definition). The existence of such a zero of $\eta_{k}$ for every $k \in\left(0, \tau_{+}(g, \sigma)\right)$ follows from [Tai92a, Tai92b, Tai93 or from [CMP04, Appendix C]. Without loss of generality we might suppose that $\alpha_{k}$ is non-contractible, as otherwise the proof contained in AB15. would go through without any change. Observe that, if $\alpha_{k}$ is non-contractible, then also its iterates $\alpha_{k}^{n}$ are noncontractible and are contained in distinct connected components, say $\mathcal{N}^{n}$, of the space $\mathcal{M}$. We use the $\alpha_{k}^{n}$ 's to define suitable minimax classes in each $\mathcal{N}^{n}$ and corresponding monotonically increasing minimax functions $c_{n}(k)$. The monotonicity will be an essential ingredient to show that the minimax functions actually yield zeros $\gamma_{n}(k)$ of $\eta_{k}$ for almost every $k \in\left(0, \tau_{+}(g, \sigma)\right)$. This is the content of the Struwe monotonicity argument [Str90], which we generalize to our setting. The fact that the sets $\mathcal{N}^{n}$ are all distinct combined with the fact that isolated zeros for $\eta_{k}$ cease to be of mountain-pass type if iterated sufficiently many times (cf. AMMP14, Theorem 2.6] or Proposition [3.1) will show that the $\gamma_{n}(k)$ 's can not be iterates of finitely many zeros of $\eta_{k}$, thus concluding the proof.

We end this introduction with a summary of the contents of the present work:

- In Section 2 we introduce the action form $\eta_{k}$ and recall its global properties.

- In Section 3 we analyze the behavior of $\eta_{k}$ locally around a zero.

- In Section 4 we recall the existence, for every sufficiently low energy, of closed magnetic geodesics which are local minimizers of the action.

- In Section 5 we define the minimax classes and the minimax functions.

- In Section 6 we prove the mountain pass lemma.

- In Section 7 we conclude the proof of the main theorem.

Acknowledgements. Luca Asselle is partially supported by the DFG grant AB 360/2-1 "Periodic orbits of conservative systems below the Mañé critical energy value". Gabriele Benedetti is partially supported by the DFG grant SFB 878. We are indebted to Marco Mazzucchelli for the notion of essential family. We would like to thank the anonymous referee for her careful reading of the draft and for her suggestions which helped us improving the paper.

\section{THE ACTION 1-FORM}

We start by defining the 1 -form $\eta_{k}$ and recalling some general facts about it. For the proofs of the statements in this section we refer to [AB16, Section 2]. Let $(M, g)$ be a closed connected Riemannian manifold and let $\sigma$ be a (closed) 2 -form on $M$. We will write $|\cdot|$ for the norm on $T M$ induced by $g$. Let us denote by $\mathcal{M}:=H^{1}(\mathbb{T}, M) \times(0,+\infty)$ the space of $H^{1}$-loops in $M$ with arbitrary period. We write $\mathcal{M}_{0}$ for the component of contractible

\footnotetext{
${ }^{1}$ See [AMMP14, Con06, Mer10] for other applications of this argument to the existence of closed orbits and Ass15] for an application to the existence of orbits satisfying the conormal boundary conditions.
} 
loops. The space $\mathcal{M}$ has the structure of a Hilbert manifold endowed with a metric given by $g_{\mathcal{M}}=g_{H^{1}}+d T^{2}$, where $g_{H^{1}}$ is the usual Riemannian metric on $H^{1}(\mathbb{T}, M)$ induced by the metric $g$. With slight abuse of notation we will also denote the norm on $\mathcal{M}$ induced by $g_{\mathcal{M}}$ with $|\cdot|$.

Throughout this paper we will adopt the identification $\gamma=(x, T)$ where $\gamma: \mathbb{R} \rightarrow M$ is such that $\gamma(t)=x(t / T)$. We have a $\mathbb{T}$ - and an $\mathbb{N}$-action on $\mathcal{M}$, where by $\mathbb{N}$ we denote the set of positive integers. The former action changes the base point of the loop:

$$
\psi \cdot \gamma:=(x(\psi+\cdot), T), \quad \forall \psi \in \mathbb{T}, \forall \gamma=(x, T) \in \mathcal{M} .
$$

The latter action iterates the loop:

$$
\gamma^{n}:=\left(x^{n}, n T\right), \quad \forall n \in \mathbb{N}, \forall \gamma=(x, T) \in \mathcal{M},
$$

where $x^{n}(s):=x(n s), \forall s \in \mathbb{T}$.

Let us now define for every $k \in(0,+\infty)$ the action 1 -form $\eta_{k} \in \Omega^{1}(\mathcal{M})$ by

$$
\eta_{k}(x, T):=d \mathbb{A}_{k}(x, T)+\int_{0}^{1} \sigma_{x(s)}\left(\cdot, x^{\prime}(s)\right) d s,
$$

where $\mathbb{A}_{k}: \mathcal{M} \rightarrow \mathbb{R}$ is given by

$$
\mathbb{A}_{k}(x, T):=T \cdot \int_{0}^{1}\left(\frac{1}{2 T^{2}}\left|x^{\prime}(s)\right|^{2}+k\right) d s=\frac{e(x)}{T}+k T
$$

and $e(x)$ is the kinetic energy of $x$

$$
e(x):=\frac{1}{2} \int_{0}^{1}\left|x^{\prime}(s)\right|^{2} d s .
$$

The action 1-form is smooth and closed. It is, furthermore, $\mathbb{T}$-invariant. In particular, the set of zeros of $\eta_{k}$ is the disjoint union of sets of the type $\mathbb{T} \cdot \gamma$. We call every such a set a vanishing circle. Moreover, an element $\gamma \in \mathcal{M}$ is a zero of $\eta_{k}$ if and only if $\gamma$ is a closed magnetic geodesic with energy $k$. In view of this, we will find closed magnetic geodesics with energy $k$ by constructing zeros of $\eta_{k}$. We will achieve this goal using an approximation procedure.

Definition 2.1. We call $\left(\gamma_{h}\right)=\left(x_{h}, T_{h}\right) \subset \mathcal{M}$ a vanishing sequence for $\eta_{k}$, if

$$
\left|\eta_{k}\left(\gamma_{h}\right)\right| \rightarrow 0 \text {. }
$$

By continuity $\eta_{k}$ vanishes on the set of limit points of vanishing sequences. So we are led to ask: which vanishing sequences do have a non-empty limit set? Clearly, if $T_{h} \rightarrow 0$ or $T_{h} \rightarrow \infty$, then the limit set is empty. The following theorem shows that the converse is also true.

Theorem 2.2. Let $\left(\gamma_{h}\right)=\left(x_{h}, T_{h}\right)$ be a vanishing sequence for $\eta_{k}$ in a given connected component of $\mathcal{M}$ with $T_{h} \leq T^{*}<\infty$ for every $h \in \mathbb{N}$. Then the following statements hold:

(1) If $T_{h}$ tends to zero, then $e\left(x_{h}\right) \rightarrow 0$.

(2) If $0<T_{*} \leq T_{h}, \forall h \in \mathbb{N}$, then $\left(\gamma_{h}\right)$ has a converging subsequence.

We are going to find vanishing sequences by considering certain minimax classes of paths in $\mathcal{M}$. For our argument we need a vector field $X_{k}$ generalizing the negative gradient of the free-period action functional. It is defined by

$$
X_{k}:=\frac{-\sharp \eta_{k}}{\sqrt{1+\left|\eta_{k}\right|^{2}}}
$$

where $\sharp$ is the duality between $T \mathcal{M}$ and $T^{*} \mathcal{M}$ induced by the Riemannian metric $g_{\mathcal{M}}$. Let $\Phi^{k}$ be the positive semi-flow of $X_{k}$. It is known that the flow lines of $\Phi^{k}$ that blow up in 
finite time go closer and closer to the subset of constant loops. Hence, the restriction of the semi-flow $\Phi^{k}$ to any connected component $\mathcal{N} \neq \mathcal{M}_{0}$ of $\mathcal{M}$ is complete, namely all its trajectories are defined for all positive times. Moreover, by the definition of $X_{k}$ we have the following consequence of Theorem 2.2.

Corollary 2.3. Let $\mathcal{N} \neq \mathcal{M}_{0}$ be a connected component of $\mathcal{M}, T^{*}$ a positive real number, and $\mathcal{Y}^{\prime} \subset \mathcal{N}$ a neighborhood of the zeros of $\eta_{k}$ that are contained in the set $\mathcal{N} \cap\left\{T \leq T^{*}\right\}$. There exists $\varepsilon=\varepsilon\left(T^{*}, \mathcal{Y}^{\prime}\right)>0$ such that

$$
\left(\mathcal{N} \cap\left\{T \leq T^{*}\right\}\right) \backslash \mathcal{Y}^{\prime} \subset\left\{\left|\eta_{k}\left(X_{k}\right)\right| \geq \varepsilon\right\} .
$$

\section{Local PROperties of THE ACTION 1-FORM ON SURFACES}

In this section we analyze some local properties of the action 1-form $\eta_{k}$ under the assumption that $M$ is a closed connected orientable surface.

If $\gamma$ is a zero of $\eta_{k}$ we now construct a neighborhood of $\mathbb{T} \cdot \gamma^{\mathbb{N}}$ where the action form admits a well-behaved primitive. Since $\gamma$ is a smooth curve, $\gamma(\mathbb{R}) \subseteq M$ is a set of zero Lebesgue measure. In particular, there exists $q \in M \backslash \gamma(\mathbb{R})$. We set $V^{\gamma}:=M \backslash\{q\}$ and observe that $\left.\sigma\right|_{V^{\gamma}}$ is exact. Let $\vartheta^{\gamma} \in \Omega^{1}\left(V^{\gamma}\right)$ be a primitive of $\sigma$ on $V^{\gamma}$. We denote by $\mathcal{V}^{\gamma}$ the open subset of $\mathcal{M}$ made by the loops with image entirely contained in $V^{\gamma}$ and observe that $\mathcal{V}^{\gamma}$ is an open neighborhood of the set $\mathbb{T} \cdot \gamma^{\mathbb{N}}$. Furthermore, $\eta_{k}$ is exact on $\mathcal{V}^{\gamma}$ for every $k \in(0,+\infty)$ with primitive $\mathbb{S}_{k}^{\gamma}: \mathcal{V}^{\gamma} \rightarrow \mathbb{R}$ given by the formula

$$
\mathbb{S}_{k}^{\gamma}(x, T):=T \cdot \int_{0}^{1}\left[L_{\vartheta \gamma}\left(x(s), \frac{x^{\prime}(s)}{T}\right)+k\right] d s, \quad L_{\vartheta \gamma}(q, v):=\frac{1}{2}|v|_{q}^{2}+\vartheta_{q}^{\gamma}(v) .
$$

Namely, $\mathbb{S}_{k}^{\gamma}$ is the free-period action functional associated with the Lagrangian $L_{\vartheta \gamma}$. Since $\mathbb{S}_{k}^{\gamma}$ belongs to the class of functionals considered in [AMMP14, we can translate Theorem 2.6 contained therein to our setting. Notice indeed that the base manifold does not have to be compact for that result to hold.

Proposition 3.1. Let $k>0$ and let $\gamma \in \mathcal{M}$ be such that for every $\nu \in \mathbb{N}, \mathbb{T} \cdot \gamma^{\nu}$ is an isolated vanishing circle. Let $\mathbb{S}_{k}^{\gamma}: \mathcal{V}^{\gamma} \rightarrow \mathbb{R}$ be the local primitive of $\eta_{k}$ defined above. There exists $\nu(\gamma) \in \mathbb{N}$ such that for all $\nu>\nu(\gamma)$ the following holds: There exists a fundamental system of open neighborhoods $\mathcal{W}_{\nu} \subseteq \mathcal{V}^{\gamma}$ of $\mathbb{T} \cdot \gamma^{\nu}$ such that, if $\beta_{0}, \beta_{1} \in\left\{\mathbb{S}_{k}^{\gamma}<\mathbb{S}_{k}^{\gamma}\left(\gamma^{\nu}\right)\right\} \cap \mathcal{W}_{\nu}$ are contained in the same connected component of $\mathcal{W}_{\nu}$ then $\beta_{0}$ and $\beta_{1}$ are contained in the same connected component of $\left\{\mathbb{S}_{k}^{\gamma}<\mathbb{S}_{k}^{\gamma}\left(\gamma^{\nu}\right)\right\}$.

Remark 3.2. Given $\beta_{0}$ and $\beta_{1}$ as above, we stress that the path connecting them inside $\left\{\mathbb{S}_{k}^{\gamma}<\mathbb{S}_{k}^{\gamma}\left(\gamma^{\nu}\right)\right\}$ might not be contained in $\mathcal{W}_{\nu}$.

We now move to consider zeros of $\eta_{k}$ of a particular type.

Definition 3.3. We say that $\alpha \in \mathcal{M}$ is a local minimizer of the action (with energy $k$ ) if there exists an open neighborhood $\mathcal{U}^{\alpha} \subseteq \mathcal{V}^{\alpha}$ of $\mathbb{T} \cdot \alpha$ such that

$$
\mathbb{S}_{k}^{\alpha}(\gamma) \geq \mathbb{S}_{k}^{\alpha}(\alpha), \quad \forall \gamma \in \mathcal{U}^{\alpha}
$$

The local minimizer $\alpha$ is called strict, if inequality (3.1) is strict $\forall \gamma \in \mathcal{U}^{\alpha} \backslash \mathbb{T} \cdot \alpha$.

The next proposition, which follows from [AMP15, Lemma 3.1], states that the property of being a local minimizer is preserved under iterations.

Proposition 3.4. If $\alpha$ is a (strict) local minimizer of the action, then for every $n \geq 1$ the $n$-th iterate $\alpha^{n}$ is also a (strict) local minimizer of the action.

The last proposition of this section gives more information on the neighborhood $\mathcal{U}^{\alpha}$, when the minimizer $\alpha$ is strict, see [AMP15, Lemma 4.3] for the proof. 
Proposition 3.5. If $\alpha$ is a strict local minimizer of the action with energy $k$, there exists an open neighborhood $\mathcal{U}^{\alpha}$ of $\mathbb{T} \cdot \alpha$ such that

$$
\inf _{\partial \mathcal{U}^{\alpha}} \mathbb{S}_{k}^{\alpha}>\mathbb{S}_{k}^{\alpha}(\alpha)
$$

\section{LOCAL MINIMIZERS FOR THE ACTION 1-FORM ON SURFACES}

We now recall the existence of local minimizers when $(M, g)$ is an orientable closed connected Riemannian surface and $\sigma$ is a 2 -form on it. Up to changing the orientation of $M$, we can also assume that the integral of $\sigma$ over $M$ is non-negative. Let $\mathcal{F}_{+}$be the space of positively oriented embedded surfaces in $M$ (in Tai92a, Tai92b, Tai93] Taŭmanov considers the so-called films). We remark that the elements in $\mathcal{F}_{+}$can have boundary or more than one connected component and that the empty surface $\varnothing$ also belongs to $\mathcal{F}_{+}$. If $k \in(0,+\infty)$ we consider the family of Taumanov functionals

$$
\mathcal{T}_{k}: \mathcal{F}_{+} \rightarrow \mathbb{R}, \quad \mathcal{T}_{k}(\Pi):=\sqrt{2 k} \cdot l(\partial \Pi)+\int_{\Pi} \sigma,
$$

where $l(\partial \Pi)$ denotes the length of the boundary of $\Pi$. We readily find that

$$
\mathcal{T}_{k}(\varnothing)=0, \quad \mathcal{T}_{k}(M)=\int_{M} \sigma \geq 0 .
$$

The family $k \mapsto \mathcal{T}_{k}$ is increasing and each $\mathcal{T}_{k}$ is bounded from below since

$$
\mathcal{T}_{k}(\Pi) \geq-\|\sigma\|_{\infty} \cdot \operatorname{area}_{g}(M) .
$$

We define now the value

$$
\tau_{+}(M, g, \sigma):=\sup \left\{k \mid \inf \mathcal{T}_{k}<0\right\} .
$$

The functionals $\mathcal{T}_{k}$ can be lifted to any finite cover $p^{\prime}: M^{\prime} \rightarrow M$, giving rise to the set of values $\tau_{+}\left(M^{\prime}, g, \sigma\right)$. We define the Taumanov critical value as

$$
\tau_{+}(g, \sigma):=\sup \left\{\tau_{+}\left(M^{\prime}, g, \sigma\right) \mid p^{\prime}: M^{\prime} \rightarrow M \text { finite cover }\right\} .
$$

In CMP04 it was shown that, when $\sigma=d \vartheta$ is exact, the Taumanov critical value coincides with $c_{0}\left(L_{\vartheta}\right)$, the Mañé critical value of the abelian cover of the Lagrangian $L_{\vartheta}$ as in (1.3). To our knowledge there is no such a precise characterization for a general $\sigma$. However, a finite upper bound for $\tau_{+}(g, \sigma)$ in terms of suitable Mañé critical values can still be found and $\tau_{+}(g, \sigma)$ turns out to be strictly positive if and only if $\sigma$ is oscillating (see AB15, Lemma 6.4] for a proof of the latter property). As far as the first assertion is concerned, let us consider any 2-form $\sigma^{\prime}$ on $M$ such that $\sigma^{\prime} \geq 0$ everywhere and the difference $\sigma-\sigma^{\prime}$ is exact. If $\vartheta$ is any primitive of $\sigma-\sigma^{\prime}$, we readily observe that

$$
\mathcal{T}_{k}(\Pi)=\sqrt{2 k} \cdot l(\partial \Pi)+\int_{\Pi} d \vartheta+\int_{\Pi} \sigma^{\prime} \geq \sqrt{2 k} \cdot l(\partial \Pi)+\int_{\Pi} d \vartheta
$$

From this inequality and from the characterization of $\tau_{+}(g, \sigma)$ in the exact case, it follows that $\tau_{+}(g, \sigma) \leq c_{0}\left(L_{\vartheta}\right)$. Thus, we conclude that $\tau_{+}(g, \sigma)$ is finite and, more precisely,

$$
\tau_{+}(g, \sigma) \leq \inf _{\sigma^{\prime}} \inf _{d \vartheta=\sigma-\sigma^{\prime}} c_{0}\left(L_{\vartheta}\right),
$$

where $\sigma^{\prime}$ is a 2 -form on $M$ such that $\sigma^{\prime} \geq 0$ and $\sigma-\sigma^{\prime}$ is exact.

We can now state the main theorem about the existence of local minimizers of the action. This result is an easy corollary of Taümanov's theorem Tai92b, Tai93, CMP04 about the existence of global minimizers for $\mathcal{T}_{k}$ and Section 3 in AMP15. For a detailed discussion when $M$ has genus at least 2, we refer to [AB15, Section 6]. 
Theorem 4.1. Let $g$ be a Riemannian metric on a closed connected orientable surface $M$ and $\sigma \in \Omega^{2}(M)$ an oscillating form. For every $k \in\left(0, \tau_{+}(g, \sigma)\right)$ there exists a closed magnetic geodesic $\alpha_{k}$ on $M$ with energy $k$ which is a local minimizer of the action.

\section{The Minimax Classes}

From now on let $\mathbb{T}^{2}$ be the two-torus endowed with a Riemannian metric $g$ and an oscillating 2-form $\sigma$. For the rest of the paper we fix $k^{*} \in\left(0, \tau_{+}(g, \sigma)\right)$. By Theorem 4.1, there exists a local minimizer with energy $k^{*}$, which we denote by $\alpha_{k^{*}}$. We notice that, if $\alpha_{k^{*}}$ is not strict, then there exists a sequence of local minimizers of the action approaching $\alpha_{k^{*}}$, which are all closed magnetic geodesics with energy $k^{*}$. Thus, from now on we suppose that the local minimizer $\alpha_{k^{*}}$ is strict. To prove Theorem 1.2 we need only show the following

Proposition 5.1. There exists an open interval $I=I\left(k^{*}\right) \subset\left(0, \tau_{+}(g, \sigma)\right)$ containing $k^{*}$ such that for almost every $k \in I$, there exist infinitely many geometrically distinct closed magnetic geodesics with energy $k$.

The proof of the proposition will occupy us for the remaining of the paper.

If $\alpha_{k^{*}}$ is contractible then we restrict the study to $\mathcal{M}_{0}$. Here, the action 1 -form $\eta_{k}$ becomes exact since $\pi_{2}\left(\mathbb{T}^{2}\right)=0$ (cf. Mer10] ) with primitive given by

$$
\mathbb{A}_{k}(x, T)+\int_{C(x)} \sigma
$$

where $C(x)$ is any capping disc for $x$. In this case, Proposition 5.1 follows by reproducing the same argument as in AB15, having in mind the compactness stated in Theorem 2.2.

Hence, hereafter we assume that $\alpha_{k^{*}}$ is non-contractible and for every $n \in \mathbb{N}$ we denote by $\mathcal{N}^{n}$ the connected component of $\mathcal{M}$ containing $\alpha_{k^{*}}^{n}$. Recall that by Proposition 3.5 we can find a bounded open neighborhood $\mathcal{U}=\mathcal{U}^{\alpha_{k^{*}}} \subseteq \mathcal{V}^{\alpha_{k^{*}}}$ of $\mathbb{T} \cdot \alpha_{k^{*}}$ such that

$$
\inf _{\partial \mathcal{U}} \mathbb{S}_{k^{*}}^{\alpha_{k^{*}}}>\mathbb{S}_{k^{*}}^{\alpha_{k^{*}}}\left(\alpha_{k^{*}}\right)
$$

Here $\mathcal{V}^{\alpha_{k^{*}}}$ is the open neighborhood of $\mathbb{T} \cdot \alpha_{k^{*}}^{\mathbb{N}}$ and $\mathbb{S}_{k^{*}}^{\alpha^{*}}$ is the local primitive of $\eta_{k^{*}}$ defined at the beginning of Section [3. For all $k \in\left(0, \tau_{+}(g, \sigma)\right)$ we define

$$
M_{k}:=\overline{\left\{\text { local minimizers of } \mathbb{S}_{k}^{\alpha_{k^{*}}} \text { in } \mathcal{U}\right\}} \text {. }
$$

We now find an interval of energies around $k^{*}$ where the sets $M_{k}$ have a good behavior. We refer to [AMMP14, Lemma 3.1 and 3.2] for the proof.

Lemma 5.2. There exists an open interval $I=I\left(k^{*}\right) \subset\left(0, \tau_{+}(g, \sigma)\right)$ containing $k^{*}$ and with the following properties:

(i) For every $k \in I$ the set $M_{k}$ is non-empty and compact.

(ii) For every pair $k_{-}<k_{+} \in I$ and for every $\beta \in M_{k_{+}}$there exists a continuous path $w:[0,1] \rightarrow \mathcal{U}$ such that $w(0) \in M_{k_{-}}, w(1)=\beta$ and

$$
\mathbb{S}_{k_{-}}^{\alpha_{k^{*}}} \circ w \leq \mathbb{S}_{k_{-}}^{\alpha_{k^{*}}}(\beta)
$$

Before defining the minimax classes we need some information about the topology of $\mathcal{M}$, which we now recall. For every $(a, b) \in \mathbb{Z}^{2}$ denote by $\mathcal{M}(a, b)$ the connected component of the loops $\gamma$ such that $\widetilde{\gamma}(T)=(a, b)+\widetilde{\gamma}(0)$, where $T$ is the period of $\gamma$ and $\widetilde{\gamma}$ is any lift of $\gamma$ to $\mathbb{R}^{2}$. Let $\mathcal{M}(a, b)^{n}$ be the connected component of $\mathcal{M}$ containing the $n$-th iterates of the elements in $\mathcal{M}(a, b)$. Since clearly $\mathcal{M}(a, b)^{n}=\mathcal{M}(n a, n b)$, the sets $\mathcal{M}(a, b)^{n}$ are pairwise disjoint for $(a, b) \neq(0,0)$. We set $\gamma_{(a, b)} \in \mathcal{M}(a, b)$ for every $(a, b) \in \mathbb{Z}^{2}$ to be the projection to $\mathbb{T}^{2}$ of the path in $\mathbb{R}^{2}$ given by $t \mapsto(t a / T, t b / T)$. It is a classical fact that

$$
\varphi_{(a, b)}: \mathbb{T}^{2} \rightarrow \mathcal{M}(a, b), \quad \varphi_{(a, b)}(q):=q+\gamma_{(a, b)}
$$


is a homotopy equivalence whose homotopy inverse is the evaluation at zero.

Let now $\bar{\sigma} \in \Omega^{2}\left(\mathbb{T}^{2}\right)$ be given by $\bar{\sigma}=d q^{1} \wedge d q^{2}$, where $\left(q^{1}, q^{2}\right)$ are the Cartesian coordinates in $\mathbb{R}^{2}$. We can associate to it the transgression 1 -form $\tau \in \Omega^{1}(\mathcal{M})$ given by

$$
\tau_{\gamma}:=\int_{0}^{T} \bar{\sigma}_{\gamma(t)}(\cdot, \dot{\gamma}(t)) d t, \quad \gamma \in \mathcal{M}
$$

Such a form is closed and, hence, it yields a cohomology class $[\tau] \in H^{1}(\mathcal{M}, \mathbb{R})$.

Lemma 5.3. Let $(a, b) \in \mathbb{Z}^{2}$, then:

(i) We have

$$
\left[\varphi_{(a, b)}^{*} \tau\right]=-\left[\imath_{(a, b)} \bar{\sigma}\right]=\left[b d q^{1}-a d q^{2}\right] \in H^{1}\left(\mathbb{T}^{2}, \mathbb{R}\right) .
$$

Thus, the restricted class $\left.[\tau]\right|_{\mathcal{M}(a, b)}$ is trivial if and only if $(a, b)=(0,0)$.

(ii) For every closed magnetic geodesic $\gamma$ the restriction $\left.[\tau]\right|_{\mathcal{V}^{\gamma}}$ is trivial, where $\mathcal{V}^{\gamma}$ is the neighborhood of $\mathbb{T} \cdot \gamma^{\mathbb{N}}$ defined in Section 3 .

Proof. Let $(q, v) \in T \mathbb{T}^{2} \simeq \mathbb{T}^{2} \times \mathbb{R}^{2}$ and compute

$$
\tau_{\varphi_{(a, b)}(q)}\left(d_{q} \varphi_{(a, b)}(v)\right)=\int_{0}^{T} \bar{\sigma}\left(v, \frac{1}{T}(a, b)\right) d t=\bar{\sigma}(v,(a, b)),
$$

which yields at once (5.3). As far as the second statement is concerned, if $\bar{\vartheta} \gamma \in \Omega^{1}\left(V^{\gamma}\right)$ is a primitive of $\left.\bar{\sigma}\right|_{V^{\gamma}}$, then a primitive of $\tau$ on $\mathcal{V}^{\gamma}$ is given by

$$
\beta \mapsto \int_{0}^{T} \beta^{*} \bar{\vartheta}^{\gamma}
$$

Corollary 5.4. For any $k \in(0,+\infty)$ and $(a, b) \in \mathbb{Z}^{2}$ we have

$$
\left[\varphi_{(a, b)}^{*} \eta_{k}\right]=\left(\int_{\mathbb{T}^{2}} \sigma\right) \cdot\left[b d q^{1}-a d q^{2}\right] \in H^{1}\left(\mathbb{T}^{2}, \mathbb{R}\right) .
$$

In particular, the image $\left[\eta_{k}\right]\left(H_{1}(\mathcal{M}(a, b), \mathbb{Z})\right)$ is a discrete subgroup of $\mathbb{R}$.

We can now proceed to the definition of the sequence of minimax classes we are interested in. For every $k \in I$ and $n \in \mathbb{N}$ we set

$$
\mathcal{P}_{n}(k):=\left\{u:[0,1] \rightarrow \mathcal{N}^{n} \mid u(0)=u(1) \in M_{k}^{n}, \int_{0}^{1} u^{*} \tau \neq 0\right\},
$$

where $M_{k}^{n}$ is made of the $n$-th iterates of the elements in $M_{k}$. Since $\mathcal{N}^{n} \neq \mathcal{M}_{0}$ the set $\mathcal{P}_{n}(k)$ is non-empty by Lemma 5.3. (i). Intuitively we can think of $\mathcal{P}_{n}(k)$ as the set of loops in $\mathcal{N}^{n}$ based at some point of $M_{k}^{n}$ whose homotopy class in $\pi_{1}\left(\mathcal{N}^{n}\right)$ is not contained in the subgroup generated by the $\mathbb{T}$-action, which changes the base point of a loop. By Lemma 5.3. (ii) we have that all the elements in $\mathcal{P}_{n}(k)$ have to leave a suitable neighborhood of $M_{k}^{n}$. This last property will be crucial in the proof of Lemma 6.4.

Remark 5.5. If $n \in \mathbb{N}$ and $k_{-}<k_{+}$, then the minimax classes $\mathcal{P}_{n}\left(k_{-}\right)$and $\mathcal{P}_{n}\left(k_{+}\right)$are disjoint. However, there is a natural way to relate them. Namely, if $u_{+} \in \mathcal{P}_{n}\left(k_{+}\right)$and $\beta^{n}=u_{+}(0)=u_{+}(1)$ for some $\beta \in M_{k_{+}}$, then Lemma 5.2.(ii) yields $w:[0,1] \rightarrow \mathcal{U}$ with $w(0) \in M_{k_{-}}, w(1)=\beta$ and such that (5.2) holds. We denote by $w^{n}, \bar{w}^{n}:[0,1] \rightarrow \mathcal{U}^{n}$ the paths defined as $w^{n}(s):=(w(s))^{n}$ and $\bar{w}^{n}(s):=(w(1-s))^{n}$, for all $s \in[0,1]$. Thus, we obtain an element $u_{-} \in \mathcal{P}_{n}\left(k_{-}\right)$as $u_{-}:=w^{n} * u_{+} * \bar{w}^{n}$, where $*$ denotes the concatenation of paths. 
For every $u \in \mathcal{P}_{n}(k)$ we now set

$$
\mathbb{S}_{k}(u, s):=\mathbb{S}_{k}^{\alpha_{k^{*}}}(u(0))+\int_{0}^{s} u^{*} \eta_{k}, \quad \forall s \in[0,1]
$$

and define $c_{n}: I \rightarrow \mathbb{R}$ by

$$
c_{n}(k):=\inf _{u \in \mathcal{P}_{n}(k)} \max _{s \in[0,1]} \mathbb{S}_{k}(u, s) .
$$

The relation between the functions $\mathbb{S}$ for two different values of the energy is described in the next lemma, cf. [AB16, Lemma 4.2] for the proof.

Lemma 5.6. Let $u=(x, T):[0,1] \rightarrow \mathcal{M}$ be a continuous path such that $u(0) \in \mathcal{V}^{\alpha_{k^{*}}}$. If $k_{-}$and $k_{+}$are positive real numbers, we have

$$
\mathbb{S}_{k_{+}}(u, s)=\mathbb{S}_{k_{-}}(u, s)+\left(k_{+}-k_{-}\right) T(s), \quad \forall s \in[0,1] .
$$

An important consequence of Remark 5.5 and Lemma 5.6 is the monotonicity of the minimax functions.

Lemma 5.7. If $n \in \mathbb{N}$ and $k_{-}, k_{+} \in I$ with $k_{-}<k_{+}$, then $c_{n}\left(k_{-}\right) \leq c_{n}\left(k_{+}\right)$.

Proof. Consider $u_{+} \in \mathcal{P}_{n}\left(k_{+}\right)$and let $u_{-}=w^{n} * u_{+} * \bar{w}^{n} \in \mathcal{P}_{n}\left(k_{-}\right)$be the path defined in Remark 5.5. In particular, we have $\left.u_{-}\right|_{[1 / 3,2 / 3]}=u_{+}(3(\cdot-1 / 3))$ and, if $\beta^{n}:=w^{n}(0)=$ $w^{n}(1)=u_{+}(0)=u_{+}(1)$, then

$$
u_{-}([0,1 / 3])=w^{n}([0,1]) \subseteq\left\{\mathbb{S}_{k_{-}}^{\alpha_{k^{*}}} \leq \mathbb{S}_{k_{-}}^{\alpha_{k^{*}}}\left(\beta^{n}\right)\right\},
$$

since $w$ satisfies (5.2) and $\mathbb{S}_{k_{-}}^{\alpha_{k^{*}}}$ is $\mathbb{N}$-equivariant. Analogously,

$$
u_{-}([2 / 3,1])=\bar{w}^{n}([0,1]) \subseteq\left\{\mathbb{S}_{k_{-}}^{\alpha_{k^{*}}} \leq \mathbb{S}_{k_{-}}^{\alpha_{k^{*}}}\left(\beta^{n}\right)\right\} .
$$

Thus, if $s \in[0,1 / 3]$, (5.8) implies that

$$
\mathbb{S}_{k_{-}}\left(u_{-}, s\right)=\mathbb{S}_{k_{-}}^{\alpha_{k^{*}}}\left(u_{-}(s)\right) \leq \mathbb{S}_{k_{-}}^{\alpha_{k^{*}}}\left(\beta^{n}\right) \leq \mathbb{S}_{k_{+}}^{\alpha_{k^{*}}}\left(u_{+}(0)\right)=\mathbb{S}_{k_{+}}\left(u_{+}, 0\right)
$$

If $s \in[1 / 3,2 / 3]$, applying (5.7) we get

$$
\begin{aligned}
\mathbb{S}_{k_{-}}\left(u_{-}, s\right)=\mathbb{S}_{k_{-}}^{\alpha_{k^{*}}}\left(u_{+}(0)\right)+\int_{0}^{3(s-1 / 3)} u_{+}^{*} \eta_{k_{-}} & =\mathbb{S}_{k_{-}}\left(u_{+}, 3(s-1 / 3)\right) \\
& \leq \mathbb{S}_{k_{+}}\left(u_{+}, 3(s-1 / 3)\right) .
\end{aligned}
$$

Finally, if $s \in[2 / 3,1],(5.9)$ implies that

$$
\begin{aligned}
\mathbb{S}_{k_{-}}\left(u_{-}, s\right) & =\mathbb{S}_{k_{-}}^{\alpha_{k^{*}}}\left(u_{+}(0)\right)+\int_{0}^{1} u_{+}^{*} \eta_{k_{-}}+\int_{2 / 3}^{s} u_{-}^{*} \eta_{k_{-}} \\
& =\mathbb{S}_{k_{-}}\left(u_{+}, 1\right)+\mathbb{S}_{k_{-}}^{\alpha_{k^{*}}}\left(u_{-}(s)\right)-\mathbb{S}_{k_{-}}^{\alpha_{k^{*}}}\left(\beta^{n}\right) \\
& \leq \mathbb{S}_{k_{+}}\left(u_{+}, 1\right) .
\end{aligned}
$$

Summarizing, we have that

$$
c_{n}\left(k_{-}\right) \leq \max _{s \in[0,1]} \mathbb{S}_{k_{-}}\left(u_{-}, s\right) \leq \max _{s \in[0,1]} \mathbb{S}_{k_{+}}\left(u_{+}, s\right)
$$

and hence taking the infimum over $u_{+} \in \mathcal{P}_{n}\left(k_{+}\right)$we see that $c_{n}\left(k_{-}\right) \leq c_{n}\left(k_{+}\right)$. 


\section{Essential FAMiLies AND the MOUNTAIN-PASs LEMma}

In the previous section we have defined the minimax classes and the corresponding minimax functions. In order to relate these objects to the zeros of $\eta_{k}$, we introduce now the notion of essential family.

Definition 6.1. Let $\mathcal{E}=\cup_{j \in J} \mathbb{T} \cdot \gamma_{j}$ be a union of isolated vanishing circles for $\eta_{k}$ on $\mathcal{N}^{n}$, where $J$ is a set of indices. A separating neighborhood $\mathcal{W}=\cup_{j \in J} \mathcal{W}_{j}$ for $\mathcal{E}$ is a union of disjoint connected open sets $\mathcal{W}_{j}$ such that $\mathbb{T} \cdot \gamma_{j} \subset \mathcal{W}_{j} \subset \mathcal{V}^{\gamma_{j}}$, for all $j \in J$. We say that $\mathcal{E}$ is an essential family for $\mathcal{P}_{n}(k)$ if for all separating neighborhoods $\mathcal{W}$ for $\mathcal{E}$, there exists $u \in \mathcal{P}_{n}(k)$ and $\lambda>0$ such that

$$
\begin{array}{ll}
\text { (A) } & \{0,1\} \subset\left\{\mathbb{S}_{k}(u, \cdot) \leq c_{n}(k)-\lambda\right\}, \\
\text { (B) } & \forall s \in\left\{\mathbb{S}_{k}(u, \cdot)>c_{n}(k)-\lambda\right\}, \exists j \in J \text { such that } \\
& (\text { B1 }) u(s) \in \mathcal{W}_{j}, \quad(\text { B2 }) \quad c_{n}(k)-\mathbb{S}_{k}(u, s)=\mathbb{S}_{k}^{\gamma_{j}}\left(\gamma_{j}\right)-\mathbb{S}_{k}^{\gamma_{j}}(u(s)) .
\end{array}
$$

Remark 6.2. By the definition of $c_{n}(k)$ every essential family is non-empty.

Remark 6.3. Condition (B2) might seem a bit mysterious at first sight. However, it just says that $\gamma_{j}$ is a critical point at level $c_{n}(k)$ for the local primitive of $\eta_{k}$ defined as

$$
\mathbb{S}_{k}^{\gamma_{j}, u, s}: \mathcal{W}_{j} \rightarrow \mathbb{R}, \quad \mathbb{S}_{k}^{\gamma_{j}, u, s}(\beta):=\mathbb{S}_{k}^{\gamma_{j}}(\beta)+\left(\mathbb{S}_{k}(u, s)-\mathbb{S}_{k}^{\gamma_{j}}(u(s))\right)
$$

Namely, condition (B2) is equivalent to

$$
\left(\mathbf{B 2}^{\prime}\right) \quad \mathbb{S}_{k}^{\gamma_{j}, u, s}\left(\gamma_{j}\right)=c_{n}(k) .
$$

The quantity $\mathbb{S}_{k}^{\gamma_{j}, u, s}(\beta)$ can also be defined as the sum of $\mathbb{S}_{k}^{\alpha_{k}}(u(0))$ and the integral of $\eta_{k}$ over the concatenation of $\left.u\right|_{[0, s]}$ with any path connecting $u(s)$ to $\beta$ within $\mathcal{W}_{j}$.

We can now establish the mountain pass lemma, whose proof relies on the celebrated Struwe's monotonicity argument [Str90], and that produces a finite essential family for $\mathcal{P}_{n}(k)$, whenever $n$ and $k$ satisfy some special hypotheses described in the statement below and discussed further in Remark 6.5.

Thus, let $\alpha_{k^{*}}$ be a non-contractible strict local minimizer belonging to a connected component $\mathcal{N} \neq \mathcal{M}_{0}$ of $\mathcal{M}$. Let $\mathcal{U} \subset \mathcal{V}^{\alpha_{k^{*}}}$ be a bounded neighborhood of $\mathbb{T} \cdot \alpha_{k^{*}}$ satisfying (3.2) and let $I \subset\left(0, \tau_{+}(g, \sigma)\right)$ be the open interval containing $k^{*}$ given by Lemma 5.2. For every $k \in I$, let $M_{k}$ be the subset of $\mathcal{U}$ as in (5.1), $\mathcal{P}_{n}(k)$ be the minimax class as in (5.5) and $c_{n}(k)$ be the minimax value as in (5.6).

Lemma 6.4. Let $n \in \mathbb{N}$ and $k \in I$ be such that $c_{n}: I \rightarrow \mathbb{R}$ is differentiable at $k$. Let $T_{*}>0$ be a Lipschitz constant for $c_{n}$ at $k$ such that

$$
T_{*}>n \cdot \sup _{\mathcal{U}} T-2
$$

and such that the vanishing circles for $\eta_{k}$ contained in $\mathcal{N}^{n} \cap\left\{T \leq T_{*}+3\right\}$ form a discrete set. If we call $\mathcal{E}_{n}(k)$ the union of such vanishing circles, then $\mathcal{E}_{n}(k)$ is essential for $\mathcal{P}_{n}(k)$.

Remark 6.5. The hypotheses that we put on $n$ and $k$ are very natural in view of Proposition 5.1. First, by the Lebesgue differentiation theorem the function $c_{n}: I \rightarrow \mathbb{R}$ is differentiable almost everywhere since it is monotone by Lemma 5.7. Second, if $\mathcal{N}^{n} \cap\left\{T \leq T_{*}+3\right\}$ contains infinitely many vanishing circles, then the existence of infinitely many geometrically distinct closed magnetic geodesics with energy $k$ trivially follows.

Proof. We divide the argument into four steps. 
Step 1. Choose a strictly decreasing sequence $k_{h} \downarrow k$ and set $\lambda_{h}:=k_{h}-k$. Without loss of generality we may suppose that for all $h \in \mathbb{N}$, there holds

$$
c_{n}\left(k_{h}\right)-c_{n}(k) \leq T_{*} \lambda_{h} .
$$

For every $h \in \mathbb{N}$ choose $u_{h}=\left(x_{h}, T_{h}\right) \in \mathcal{P}_{n}\left(k_{h}\right)$ such that

$$
\max _{s \in[0,1]} \mathbb{S}_{k_{h}}\left(u_{h}, s\right)<c_{n}\left(k_{h}\right)+\lambda_{h} .
$$

Let $s \in[0,1]$ be such that $\mathbb{S}_{k}\left(u_{h}, s\right)>c_{n}(k)-\lambda_{h}$. It follows

$$
T_{h}(s)=\frac{\mathbb{S}_{k_{h}}\left(u_{h}, s\right)-\mathbb{S}_{k}\left(u_{h}, s\right)}{\lambda_{h}}<\frac{\left(c_{n}\left(k_{h}\right)+\lambda_{h}\right)-\left(c_{n}(k)-\lambda_{h}\right)}{\lambda_{h}} \leq T_{*}+2
$$

and at the same time, using (6.2),

$$
\mathbb{S}_{k}\left(u_{h}, s\right) \leq \mathbb{S}_{k_{h}}\left(u_{h}, s\right)<c_{n}(k)+\left(T_{*}+1\right) \lambda_{h} .
$$

Thus, $\forall h \in \mathbb{N}$ and $\forall s \in[0,1]$ one between conditions $(a)$ and (b) below holds:

$$
\begin{aligned}
& \text { (a) } \mathbb{S}_{k}\left(u_{h}, s\right) \leq c_{n}(k)-\lambda_{h}, \\
& \text { (b) } \mathbb{S}_{k}\left(u_{h}, s\right) \in\left(c_{n}(k)-\lambda_{h}, c_{n}(k)+\left(T_{*}+1\right) \lambda_{h}\right), \quad T_{h}(s)<T_{*}+2 .
\end{aligned}
$$

By Remark [5.5] applied to $k_{-}=k$ and $k_{+}=k_{h}$, we can form the concatenated paths $w_{h}^{n} * u_{h} * \bar{w}_{h}^{n}$, which belong to $\mathcal{P}_{n}(k)$. Thanks to (6.1) and the properties of $w_{h}$ prescribed by Lemma [5.2. (ii), $w_{h}^{n} * u_{h} * \bar{w}_{h}^{n}$ satisfy the above dichotomy, as well. By a slight abuse of notation we will also denote such paths by $u_{h}$, so that, from now on, $u_{h}$ will be an element of $\mathcal{P}_{n}(k)$. Moreover, by (6.1) the set $M_{k}$ is the union of a finite number of vanishing circles, which are then strict local minimizers of the action. Up to taking a subsequence and using the $\mathbb{T}$-action on loops, we may also suppose that all the paths $u_{h}$ start from and end at the same strict local minimizer $\xi \in M_{k}^{n}$.

Step 2. Let $\Phi^{k}$ denote the semi-flow of the bounded vector field $X_{k}$ conformally equivalent to $-\sharp \eta_{k}$ defined in (2.1). As we saw in Section 2, the restriction of such a semi-flow to $\mathcal{N}^{n}$ is complete since $\mathcal{N}^{n} \neq \mathcal{M}_{0}$. Hence, we can consider for every $r \in[0,1]$ the element $u_{h}^{r} \in \mathcal{P}_{n}(k)$ given by

$$
u_{h}^{r}(s):=\Phi_{r}^{k}\left(u_{h}(s)\right), \quad \forall s \in[0,1] .
$$

Since $\eta_{k}$ is a closed form, for all $r, s \in[0,1]$, Stokes' Theorem yields the relation

$$
\mathbb{S}_{k}\left(u_{h}^{r}, s\right)=\mathbb{S}_{k}\left(u_{h}, s\right)+\int_{0}^{r} \eta_{k}\left(\frac{\partial}{\partial r^{\prime}} u_{h}^{r^{\prime}}(s)\right) d r^{\prime} .
$$

As $\frac{\partial}{\partial r^{\prime}} u_{h}^{r^{\prime}}(s)=X_{k}$, the second term on the right is non-positive. Hence, the map $r^{\prime} \mapsto$ $\mathbb{S}_{k}\left(u_{h}^{r^{\prime}}, s\right)$ is decreasing. Combining this fact with (6.3) and (6.4), we see that, for all $s \in[0,1]$, one between conditions $\left(a^{\prime}\right)$ and $\left(b^{\prime}\right)$ below holds:

$$
\begin{array}{ll}
\left(a^{\prime}\right) & \mathbb{S}_{k}\left(u_{h}^{1}, s\right) \leq c_{n}(k)-\lambda_{h}, \\
\left(b^{\prime}\right) & \mathbb{S}_{k}\left(u_{h}^{r}, s\right) \in\left(c_{n}(k)-\lambda_{h}, c_{n}(k)+\left(T_{*}+1\right) \lambda_{h}\right), \quad \forall r \in[0,1] .
\end{array}
$$

Suppose that $s \in[0,1]$ satisfies the second alternative. We get that

$$
\mathbb{S}_{k}\left(u_{h}^{r}, s\right)-\mathbb{S}_{k}\left(u_{h}, s\right)>\left(c_{n}(k)-\lambda_{h}\right)-\left(c_{n}(k)+\left(T_{*}+1\right) \lambda_{h}\right)=-\left(T_{*}+2\right) \lambda_{h} .
$$

This inequality enables us to bound the variation of the period along a flow line. Indeed, in general, it holds

$$
\left|T_{h}^{r}(s)-T_{h}(s)\right|^{2} \leq r \cdot\left(-\int_{0}^{r} \eta_{k}\left(\frac{\partial}{\partial r^{\prime}} u_{h}^{r^{\prime}}(s)\right) d r^{\prime}\right), \quad \forall r \in[0,1] .
$$


Combining this estimate with (6.5) and (6.6), we obtain

$$
T_{h}^{r}(s) \leq\left|T_{h}^{r}(s)-T_{h}(s)\right|+T_{h}(s) \leq \sqrt{r\left(T_{*}+2\right) \lambda_{h}}+\left(T_{*}+2\right)<T_{*}+3,
$$

where the last inequality is true for $h$ sufficiently large.

Step 3. We claim that for every neighborhood $\mathcal{Y}$ of the family $\mathcal{E}_{n}(k)$ and for all $h$ sufficiently large, the following implication holds:

$$
\forall s \in[0,1], \quad \mathbb{S}_{k}\left(u_{h}^{1}, s\right)>c_{n}(k)-\lambda_{h} \quad \Longrightarrow \quad u_{h}^{1}(s) \in \mathcal{Y} .
$$

Consider a neighborhood $\mathcal{Y}^{\prime} \subset \mathcal{Y}$ of $\mathcal{E}_{n}(k)$ such that $\Phi_{r}^{k}\left(\mathcal{Y}^{\prime}\right) \subset \mathcal{Y}$ for all $r \in[0,1]$. We apply Corollary 2.3 to $T_{*}+3$ and we find $\varepsilon>0$ such that

$$
\left\{T \leq T_{*}+3\right\} \backslash \mathcal{Y}^{\prime} \subset\left\{\left|\eta_{k}\left(X_{k}\right)\right| \geq \varepsilon\right\} .
$$

Suppose that, for some $s \in[0,1]$,

$$
\mathbb{S}_{k}\left(u_{h}^{1}, s\right)>c_{n}(k)-\lambda_{h}, \quad u_{h}^{1}(s) \notin \mathcal{Y} .
$$

Then, $u_{h}^{r}(s) \in\left\{T \leq T_{*}+3\right\} \backslash \mathcal{Y}^{\prime}$ for all $r \in[0,1]$. Combining Estimate (6.8) with Equation (6.5) we find a contradiction to (6.6) for $h$ large enough:

$$
\mathbb{S}_{k}\left(u_{h}^{1}, s\right)-\mathbb{S}_{k}\left(u_{h}, s\right) \leq-\varepsilon .
$$

Step 4. Let $\mathcal{W}$ be a separating neighborhood for $\mathcal{E}_{n}(k)$. We claim that there exists $h_{\mathcal{W}}$ such that if $h \geq h_{\mathcal{W}}$, then $u_{h}^{1}, \lambda_{h}$ and $\mathcal{W}$ satisfy conditions (A) and (B) in Definition 6.1.

Let $\xi$ be the element in $M_{k}^{n}$ given by Step 1 . Since $\xi$ is a strict local minimizer, by Proposition 3.5 it has a neighborhood $\mathcal{U}^{\xi} \subset \mathcal{V}^{\alpha_{k} *}$ satisfying (3.2). By Lemma [5.3, $u_{h}^{1}([0,1])$ is not contained in $\mathcal{U}^{\xi}$ for any $h \in \mathbb{N}$. In particular, there exist a smallest $s_{h}^{-}$and a largest $s_{h}^{+}$in $[0,1]$ such that $u_{h}^{1}\left(s_{h}^{ \pm}\right) \in \partial \mathcal{U}^{\xi}$ and hence there exists $\rho>0$ such that

$$
\mathbb{S}_{k}^{\alpha_{k^{*}}}(\xi)+\rho<\mathbb{S}_{k}^{\alpha_{k^{*}}}\left(u_{h}^{1}\left(s_{h}^{ \pm}\right)\right) .
$$

Using the fact that $\max \mathbb{S}_{k}\left(u_{h}^{1}, \cdot\right) \leq c_{n}(k)+\left(T_{*}+1\right) \lambda_{h}$, we now compute

$$
\begin{aligned}
\mathbb{S}_{k}\left(u_{h}^{1}, 0\right) & =\mathbb{S}_{k}\left(u_{h}^{1}, s_{h}^{-}\right)-\int_{0}^{s_{h}^{-}}\left(u_{h}^{1}\right)^{*} \eta_{k} \\
& \leq c_{n}(k)+\left(T_{*}+1\right) \lambda_{h}+\mathbb{S}_{k}^{\alpha_{k^{*}}}(\xi)-\mathbb{S}_{k}^{\alpha_{k^{*}}}\left(u_{h}^{1}\left(s_{h}^{-}\right)\right) \\
& <c_{n}(k)+\left(T_{*}+1\right) \lambda_{h}-\rho .
\end{aligned}
$$

Analogously, we get $\mathbb{S}_{k}\left(u_{h}^{1}, 1\right)<c_{n}(k)+\left(T_{*}+1\right) \lambda_{h}-\rho$ and for $h$ large

$$
\max \left\{\mathbb{S}_{k}\left(u_{h}^{1}, 0\right), \mathbb{S}_{k}\left(u_{h}^{1}, 1\right)\right\} \leq c_{n}(k)-\lambda_{h} .
$$

Now that (A) is established, we move to consider (B1). Step 3 implies

$$
u_{h}\left(\left\{\mathbb{S}_{k}\left(u_{h}^{1}, \cdot\right)>c_{n}(k)\right\}\right) \subset \mathcal{W}, \quad \text { for } h \text { large. }
$$

To show (B2) we need to prove the following statement: Given $\mathbb{T} \cdot \gamma \in \mathcal{E}_{n}(k)$ and the connected component $\mathcal{W}_{\gamma}$ of $\mathcal{W}$ such that $\gamma \in \mathcal{W}_{\gamma}$, there holds

$$
\mathbb{S}_{k}^{\gamma, u_{h}^{1}, s_{h}}(\gamma)=c_{n}(k), \quad \forall s_{h} \in\left\{\mathbb{S}_{k}\left(u_{h}^{1}, \cdot\right)>c_{n}(k)-\lambda_{h}\right\} \cap\left(u_{h}^{1}\right)^{-1}\left(\mathcal{W}_{\gamma}\right),
$$

for $h$ large and with $\mathbb{S}^{\gamma, u_{h}^{1}, s_{h}}$ defined as in Remark 6.3. Thus, let $s_{h} \in[0,1]$ be such that $\mathbb{S}_{k}\left(u_{h}^{1}, s_{h}\right)>c_{n}(k)-\lambda_{h}$ and $u_{h}^{1}\left(s_{h}\right) \in \mathcal{W}_{\gamma}$. We set

$$
a_{h}:=\mathbb{S}_{k}^{\gamma, u_{h}^{1}, s_{h}}(\gamma) \text {. }
$$

First, we claim that there is $\delta>0$ such that if $a_{h} \neq a_{h^{\prime}}$, for $h, h^{\prime} \in \mathbb{N}$, then

$$
\left|a_{h}-a_{h^{\prime}}\right| \geq \delta \text {. }
$$


Indeed, thanks to Remark 6.3, there exists $v_{h, h^{\prime}}: \mathbb{T} \rightarrow \mathcal{N}^{n}$ such that

$$
a_{h}-a_{h^{\prime}}=\int_{\mathbb{T}} v_{h, h^{\prime}}^{*} \eta_{k}
$$

The path $v_{h, h^{\prime}}$ is the concatenation of four paths: the restriction $\left.u_{h}^{1}\right|_{\left[0, s_{h}\right]}$, a path connecting $u_{h}^{1}\left(s_{h}\right)$ to $\gamma_{j}$ inside $\mathcal{W}_{j}$, a path connecting $\gamma_{j}$ to $u_{h^{\prime}}^{1}\left(s_{h^{\prime}}\right)$ inside $\mathcal{W}_{j}$ and the restriction $\left.u_{h^{\prime}}^{1}\right|_{\left[0, s_{h^{\prime}}\right]}$ traversed in the opposite direction. The claim then follows from Corollary [5.4, Thus, if we prove that $a_{h} \rightarrow c_{n}(k)$, we find $a_{h}=c_{n}(k)$ for $h$ large, as we wanted. By definition we have

$$
a_{h}-c_{n}(k)=\left(\mathbb{S}_{k}\left(u_{h}^{1}, s_{h}\right)-c_{n}(k)\right)+\left(\mathbb{S}_{k}^{\gamma}(\gamma)-\mathbb{S}_{k}^{\gamma}\left(u_{h}^{1}\left(s_{h}\right)\right)\right) .
$$

The first term on the right tends to zero since $\left|\mathbb{S}_{k}\left(u_{h}^{1}, s_{h}\right)-c_{n}(k)\right| \leq\left(T_{*}+1\right) \lambda_{h}$, whereas the second term tends to zero since $\mathbb{S}_{k}^{\gamma}$ is a continuous function and $u_{h}^{1}\left(s_{h}\right) \rightarrow \gamma$. This very last statement follows by applying Step 3 to an arbitrary separating neighborhood of $\mathcal{E}_{n}(k)$. We conclude that the sequence $\left(a_{h}\right)$ tends to $c_{n}(k)$, thus proving Step 4 and the entire lemma.

\section{The elimination lemma and the proof of the Main Theorem}

In this section we prove Theorem 1.2 by establishing Proposition 5.1 through the following elimination lemma.

Lemma 7.1. Let $\mathcal{E}$ be any essential family for $\mathcal{P}_{n}(k)$ and let $\gamma \in \mathcal{M}$ be such that $\mathbb{T} \cdot \gamma^{\nu}$ is an isolated vanishing circle of $\eta_{k}$, for all $\nu \in \mathbb{N}$. Let $\nu(\gamma)$ be the positive integer defined in Proposition 3.1. If $\nu>\nu(\gamma)$, then $\mathcal{E}^{\prime}:=\mathcal{E} \backslash \mathbb{T} \cdot \gamma^{\nu}$ is also an essential family for $\mathcal{P}_{n}(k)$.

Proof. Fix $\nu>\nu(\gamma)$ and let $\mathcal{W}_{\nu} \subset \mathcal{V}^{\gamma}$ be an arbitrarily small connected neighborhood of $\mathbb{T} \cdot \gamma^{\nu}$ such that the condition given by Proposition 3.1 holds.

Let $\mathcal{W}^{\prime}$ be a separating neighborhood for $\mathcal{E}^{\prime}=\mathcal{E} \backslash \mathbb{T} \cdot \gamma^{\nu}$. Up to shrinking the neighborhoods, we can assume that $\mathcal{W}^{\prime} \cap \mathcal{W}_{\nu}=\varnothing$, so that $\mathcal{W}:=\mathcal{W}^{\prime} \cup \mathcal{W}_{\nu}$ is a separating neighborhood for $\mathcal{E}$. Since $\mathcal{E}$ is an essential family for $\mathcal{P}_{n}(k)$ there exist $u \in \mathcal{P}_{n}(k)$ and $\lambda>0$ as in Definition 6.1. We claim that there exists a finite, and possibly empty, collection $K_{1}, \ldots, K_{j_{*}}$ of closed intervals, which are pairwise disjoint, contained in $[0,1]$ and such that

$$
\begin{aligned}
& \text { (a) }\left\{\begin{array}{c}
K_{j} \subset u^{-1}\left(\mathcal{W}_{\nu}\right) \cap\left\{\mathbb{S}_{k}(u, \cdot)>c_{n}(k)-\lambda\right\} \\
\partial K_{j} \subset\left\{\mathbb{S}_{k}(u, \cdot)<c_{n}(k)\right\}
\end{array} \mid \forall j \in\left\{1, \ldots, j_{*}\right\},\right. \\
& \text { (b) }\left\{\mathbb{S}_{k}(u, \cdot)>c_{n}(k)-\lambda\right\} \backslash K \subset \mathcal{W}^{\prime}, \quad K:=K_{1} \cup \ldots \cup K_{r} .
\end{aligned}
$$

The proof of the claim goes as follows. We observe that

$$
[0,1]=\left(\left\{\mathbb{S}_{k}(u, \cdot)>c_{n}(k)-\lambda\right\} \cap u^{-1}\left(\mathcal{W}_{\gamma}\right)\right) \cup\left(\left\{\mathbb{S}_{k}(u, \cdot)<c_{n}(k)\right\} \cup u^{-1}\left(\mathcal{W}^{\prime}\right)\right) .
$$

We denote by $\left\{B_{a} \mid a \in A\right\}$ the set of connected components of the open set $\left\{\mathbb{S}_{k}(u, \cdot)>\right.$ $\left.c_{n}(k)-\lambda\right\} \cap u^{-1}\left(\mathcal{W}_{\gamma}\right) \subset(0,1)$, where $A$ is some set of indices. The $B_{a}$ 's are pairwise disjoint and each of them is an open interval. Since $[0,1]$ is compact, there exists a finite family $B_{a(1)}, \ldots, B_{a(r)}$ such that

$$
[0,1]=\left(\bigcup_{j=1}^{r} B_{a(j)}\right) \cup\left(\left\{\mathbb{S}_{k}(u, \cdot)<c_{n}(k)\right\} \cup u^{-1}\left(\mathcal{W}^{\prime}\right)\right) .
$$

The claim follows by taking $K_{j}$ to be any closed interval contained in $B_{a(j)}$ with the property that $\partial K_{j} \subset\left\{\mathbb{S}_{k}(u, \cdot)<c_{n}(k)\right\} \cup u^{-1}\left(\mathcal{W}^{\prime}\right)$. 
Now that the claim has been proved, we can apply Proposition 3.1 with $\left\{\beta_{0}, \beta_{1}\right\}=\partial K_{j}$, for all $j=1, \ldots, r$, and obtain paths $v_{j}: K_{j} \rightarrow \mathcal{V}^{\gamma}$ such that

$$
\left.v_{j}\right|_{\partial K_{j}}=\left.u\right|_{\partial K_{j}}, \quad v_{j}\left(K_{j}\right) \subset\left\{\mathbb{S}_{k}^{\gamma} \leq \mathbb{S}_{k}^{\gamma}\left(\gamma^{\nu}\right)-\lambda^{\prime}\right\},
$$

for some $\lambda^{\prime} \in(0, \lambda]$ independent of $j$. We now modify the path $u$ to a path $v:[0,1] \rightarrow \mathcal{N}^{n}$ requiring

$$
\text { (i) } v=u \text { on }[0,1] \backslash K,\left.\quad(\text { ii }) \quad v\right|_{K_{j}}=v_{j}, \quad \forall j=1, \ldots, r .
$$

As $\{0,1\} \cap K=\varnothing$, we see that $v(0)=u(0)=u(1)=v(1)$. Moreover, since $u$ and $v$ coincide on the complement of the open set $\mathcal{V}^{\gamma}$, we have

$$
\int_{0}^{1} v^{*} \tau=\int_{0}^{1} u^{*} \tau \neq 0
$$

by Lemma [5.3, so that $v \in \mathcal{P}_{n}(k)$. For the same reason $\mathbb{S}_{k}(v, \cdot)=\mathbb{S}_{k}(u, \cdot)$ on $[0,1] \backslash K$. Let now $j$ be in $\{1, \ldots, r\}$. For all $s \in K_{j}$, there holds

$$
\lambda^{\prime} \leq \mathbb{S}_{k}^{\gamma}\left(\gamma^{\nu}\right)-\mathbb{S}_{k}^{\gamma}(v(s))=c_{n}(k)-\mathbb{S}_{k}(v, s)
$$

where we used (7.1) and property (B2). This shows that

$$
K_{j} \subset\left\{\mathbb{S}_{k}(v, \cdot) \leq c_{n}(k)-\lambda^{\prime}\right\} .
$$

Applying this argument to every $j$, we conclude that

$$
\left\{\mathbb{S}_{k}(v, \cdot)>c_{n}(k)-\lambda^{\prime}\right\} \subset v^{-1}\left(\mathcal{W}^{\prime}\right) .
$$

Thus, $\mathcal{E}^{\prime}$ is also an essential family for $\mathcal{P}_{n}(k)$ and the lemma is proved.

Proof of Proposition 5.1. We only need to consider the case in which $\alpha_{k^{*}}$ belongs to a connected component $\mathcal{N}$ of $\mathcal{M}$ different from $\mathcal{M}_{0}$. We set

$$
I^{\prime}:=\bigcap_{n \in \mathbb{N}} I_{n}^{\prime}, \quad I_{n}^{\prime}:=\left\{k \in I \mid c_{n} \text { is differentiable at } k\right\} .
$$

By the Lebesgue differentiation theorem every function $c_{n}$ is differentiable almost everywhere, since $c_{n}$ is monotone by Lemma 5.6. In particular, every $I_{n}^{\prime} \subset I$ is a full-measure set. The same is true for $I^{\prime}$ being a countable intersection of full-measure sets in a space of finite measure.

We claim that for all $k \in I^{\prime}$ there exist infinitely many closed magnetic geodesics with energy $k$ in $\bigcup_{n} \mathcal{N}^{n}$. Suppose by contradiction that there exists $k \in I^{\prime}$ such that the zero-set of $\eta_{k}$ in $\bigcup_{n} \mathcal{N}^{n}$ consists of finitely many circles

$$
\mathbb{T} \cdot \beta_{1}, \ldots, \mathbb{T} \cdot \beta_{r}
$$

together with their iterates. In particular, all the vanishing circles in $\bigcup_{n} \mathcal{N}^{n}$ are isolated. We apply Proposition 3.1 to the orbits $\beta_{1}, \ldots, \beta_{r}$ and get numbers $\nu\left(\beta_{1}\right), \ldots, \nu\left(\beta_{r}\right) \in \mathbb{N}$. We define the family

$$
\mathcal{F}:=\bigcup_{1 \leq j \leq r} \bigcup_{1 \leq \nu \leq \nu\left(\beta_{j}\right)} \mathbb{T} \cdot \beta_{j}^{\nu}
$$

Since $\mathcal{N}^{n_{1}} \neq \mathcal{N}^{n_{2}}$ if $n_{1} \neq n_{2}$ and $\mathcal{F}$ is a finite union of circles, there exists $n \in \mathbb{N}$ such that $\mathcal{N}^{n} \cap \mathcal{F}=\varnothing$. By definition of $I^{\prime}$ and the fact that all vanishing circles in $\mathcal{N}^{n}$ are isolated, we can apply Lemma 6.4 and get an essential family $\mathcal{E}_{n}(k) \subset \mathcal{N}^{n}$ for $\mathcal{P}_{n}(k)$, which is a finite union of circles. By Lemma 7.1

$$
\mathcal{E}_{n}(k) \cap \mathcal{F}=\varnothing
$$

is an essential family, as well. Since an essential family cannot be empty by Remark 6.2, we get a contradiction, which proves the proposition. 


\section{REFERENCES}

$\left[\mathrm{AAB}^{+} 16\right]$ A. Abbondandolo, L. Asselle, G. Benedetti, M. Mazzucchelli, and I. A. Taimanov, The multiplicity problem for periodic orbits of magnetic flows on the 2-sphere, preprint, arXiv:1608.03144, 2016.

[AB15] L. Asselle and G. Benedetti, Infinitely many periodic orbits of non-exact oscillating magnetic flows on surfaces with genus at least two for almost every low energy level, Calc. Var. Partial Differential Equations 54 (2015), no. 2, 1525-1545, doi:10.1007/s00526-015-0834-1

[AB16] _ The Lusternik-Fet theorem for autonomous Tonelli Hamiltonian systems on twisted cotangent bundles, J. Topol. Anal. 8 (2016), no. 3, 545-570, doi/10.1142/S1793525316500205.

[Abb13] A. Abbondandolo, Lectures on the free period Lagrangian action functional, J. Fixed Point Theory Appl. 13 (2013), no. 2, 397-430.

[AM16] L. Asselle and M. Mazzucchelli, On Tonelli periodic orbits with low energy on surfaces, preprint, arXiv:1601.06692, 2016.

[AMMP14] A. Abbondandolo, L. Macarini, M. Mazzucchelli, and G. P. Paternain, Infinitely many periodic orbits of exact magnetic flows on surfaces for almost every subcritical energy level, to appear in J. Eur. Math. Soc. (JEMS), arXiv:1404.7641, 2014.

[AMP15] A. Abbondandolo, L. Macarini, and G. P. Paternain, On the existence of three closed magnetic geodesics for subcritical energies, Comment. Math. Helv. 90 (2015), no. 1, 155-193.

[Arn61] V. I. Arnol'd, Some remarks on flows of line elements and frames, Dokl. Akad. Nauk SSSR 138 (1961), 255-257.

[Ass15] L. Asselle, On the existence of Euler-Lagrange orbits satisfying the conormal boundary conditions, to appear in J. Funct. Anal., arXiv:1507.05883, 2015.

[Ben] G. Benedetti, Surfaces of section for strong magnetic fields on surfaces, in preparation.

[Ben15] Magnetic Katok examples on the two-sphere, to appear in Bull. Lond. Math. Soc., arXiv:1507.05341, 2015.

[Ben16] - The contact property for symplectic magnetic fields on $S^{2}$, Ergodic Theory Dynam. Systems 36 (2016), no. 3, 682-713, doi:10.1017/etds.2014.82

[BZ15] G. Benedetti and K. Zehmisch, On the existence of periodic orbits for magnetic systems on the two-sphere, J. Mod. Dyn. 9 (2015), no. 01, 141-146.

[CMP04] G. Contreras, L. Macarini, and G. P. Paternain, Periodic orbits for exact magnetic flows on surfaces, Int. Math. Res. Not. (2004), no. 8, 361-387.

[Con06] G. Contreras, The Palais-Smale condition on contact type energy levels for convex Lagrangian systems, Calc. Var. Partial Differential Equations 27 (2006), no. 3, 321-395.

[FH03] J. Franks and M. Handel, Periodic points of Hamiltonian surface diffeomorphisms, Geom. Topol. 7 (2003), 713-756 (electronic).

[GGM15] V. L. Ginzburg, B. Z. Gürel, and L. Macarini, On the Conley conjecture for Reeb flows, Internat. J. Math. 26 (2015), no. 7, 1550047, 22.

[Mer10] W. J. Merry, Closed orbits of a charge in a weakly exact magnetic field, Pacific J. Math. 247 (2010), no. 1, 189-212.

[Str90] M. Struwe, Existence of periodic solutions of Hamiltonian systems on almost every energy surface, Bol. Soc. Brasil. Mat. (N.S.) 20 (1990), no. 2, 49-58.

[Tai92a] I. A. Taimanov, Closed extremals on two-dimensional manifolds, Russian Math. Surveys 47 (1992), no. 2, 163-211.

[Tai92b] N Non-self-intersecting closed extremals of multivalued or not-everywhere-positive functionals, Math. USSR-Iz. 38 (1992), no. 2, 359-374.

[Tai93] _ Closed non-self-intersecting extremals of multivalued functionals, Siberian Math. J. 33 (1993), no. 4, 686-692.

[Tai15] On an integrable magnetic geodesic flow on the two-torus, Regul. Chaotic Dyn. 20 (2015), no. 6, 667-678.

Ruhr-Universität Bochum, FAkultät für Mathematik, NA 4/35, Universitätsstrasse 150, D-44780 Bochum, Germany

E-mail address: luca.asselle@ruhr-uni-bochum.de

Universität Leipzig, Mathematisches Institut, Augustusplatz 10, D-04109 Leipzig, Germany E-mail address: gabriele.benedetti@math.uni-leipzig.de 